\title{
Robust ZSM-5 Membranes for Efficient Bio-oil Dehydration: Transport Mechanism and Its Implication on Structural Tuning
}

Gang Li, ${ }^{* \dagger}$ Shanhong Ma,${ }^{\star}$ Feng Ye,${ }^{\dagger}$ Liang Zhou,,${ }^{\S}$ Yanhong Wang,${ }^{\dagger}$ Xuemei Lang, ${ }^{\dagger}$ Shuanshi $\operatorname{Fan}^{*}, \dagger$

†School of Chemistry and Chemical Engineering, South China University of Technology, Guangzhou 510641, China.

${ }^{\ddagger}$ School of Light Industry and Engineering, South China University of Technology, Guangzhou 510641, China.

${ }^{\S}$ School of Chemical Engineering, Dalian University of Technology, Panjin 124221, China.

*Corresponding authors: fegli@ scut.edu.cn (G. Li); zhouliang@ dlut.edu.cn (Li. Zhou); ssfan@scut.edu.cn (S. Fan)

Table S1. The elemental composition of bio-oil. ${ }^{\mathrm{a}}$

\begin{tabular}{lccccc}
\hline Element & $\mathrm{C}$ & $\mathrm{H}$ & $\mathrm{S}$ & $\mathrm{N}$ & $\mathrm{O}^{\mathrm{b}}$ \\
\hline Content $[\mathrm{wt} \%]$ & 53.65 & 6.364 & 0.138 & 1.41 & 39.848 \\
\hline
\end{tabular}

${ }^{a}$ On a moisture- and ash-free basis.

${ }^{\mathrm{b}}$ By difference. 
Table S2. Contents of certain compounds in bio-oil.

\begin{tabular}{lll}
\hline No. & Compound & Content [wt\%] \\
\hline 1 & Water & 40.0 \\
2 & Acetol & 2.978 \\
3 & Acetic aid & 4.269 \\
4 & 2-Cyclopentenone & 0.231 \\
5 & Propionic acid & 0.633 \\
6 & 2-Furaldehyde & 0.393 \\
7 & Butyric acid & 0.284 \\
8 & o-Cresol & 0.156 \\
9 & m-Cresol & 0.058 \\
10 & Guaiacol & 0.007 \\
11 & 2,6-Dimethylphenol & 0.002 \\
12 & 2-Methoxy-4-methylphenol & 0.032 \\
13 & Phenol & 0.002 \\
14 & 2-Ethylphenol & 0.030 \\
\hline
\end{tabular}

Table S3. Si/Al ratios of ZSM-5 crystals after being exposed to bio-oil for different times.

\begin{tabular}{lccc}
\hline Sample & As-prepared & In bio-oil for 7 days & In bio-oil for 14 days \\
\hline Si/Al ratio & 15.21 & 13.22 & 13.03 \\
\hline
\end{tabular}

